\title{
BLACK HOLES IN HIGHER DIMENSIONS (BLACK STRINGS AND BLACK RINGS)
}

\author{
JUTTA KUNZ \\ Institut für Physik, Universität Oldenburg, Postfach 2503 \\ D-26111 Oldenburg, Germany \\ E-mail: jutta.kunz@uni-oldenburg.de
}

\begin{abstract}
The main focus of this session was the presentation of new higher-dimensional black hole solutions, including black rings, black strings, and multi black holes, and the study of their properties. Besides new asymptotically flat and locally asymptotically flat black objects also new black holes with anti-de Sitter asymptotics were reported. The studies of their properties included the investigation of their stability, their thermodynamics, their analyticity and their existence. Furthermore, the geodesics in such higher-dimensional space-times were investigated.
\end{abstract}

Keywords: Black Holes, Black Rings, Black Strings

\section{Introduction}

Today string theory plays a major role as a candidate for the quantum theory of gravity and the unification of all interactions. String theory requires higher dimensions for its mathematical consistency, which presents a strong motivation to study the possible physical consequences of the presence of more than four space-time dimensions. One very active area here is the study of higher-dimensional black holes, where the extra dimensions are considered to be either infinite or compact.

Moreover, for many years now the Anti-de Sitter/conformal field theory (AdS/CFT) correspondence has been employed as a means to study strongly coupled theories, where perturbative methods are not applicable. This correspondence allows to translate dynamical problems in strongly coupled theories in $D$ dimensions into gravitational ones in $D+1$ dimensions. This implies that finding new black holes and other black objects in $D+1$ dimensions allows for the prediction of new thermal phases in the dual field theories in $D$ dimensions.

In four dimensions the stationary, asymptotically flat electrovac black holes are given by the family of Kerr-Newman solutions. The Kerr-Newman black holes are uniquely characterized by their global charges, the mass, the angular momentum and the electromagnetic charge(s). A spatial section of their event horizon has the topology of a two-sphere, $S^{2}$, other horizon topologies are not allowed.

Already in 1986 Myers and Perry ${ }^{1}$ obtained the generalizations of the fourdimensional Kerr solutions. These Myers-Perry black holes represent asymptotically flat rotating vacuum black holes in $D$ dimensions, which possess a spherical horizon topology, $S^{D-2}$. Moreover, Myers and Perry argued that also black holes with a ring topology of the horizon should exist. When the five-dimensional vacuum black ring with horizon topology $S^{1} \times S^{2}$ was found by Emparan and Reall, ${ }^{2}$ this created a surge of interest. Soon many related black objects were found in five dimensions including black saturns, black di-rings or bicycling black rings (for a review see 
e.g! $\left.!^{3}\right)$

\section{New higher-dimensional solutions}

In the following we will discuss the various types of new solutions, that were presented in the session, starting with black rings in six dimensions.

\subsection{Black rings in more than five dimensions}

While there are a number of construction methods known to obtain exact black hole solutions in five dimensions, there does not seem to exist a general analytic framework for the construction of black objects in more than five dimensions. Therefore one has to either resort to perturbative methods or to numerical methods at the moment.

Myers and Perry already suggested a heuristic way to construct black rings, namely to take a Schwarzschild black string, to bend it, and to achieve balance by spinning it along the ring $S^{1}$ direction. ${ }^{2}$ The perturbative technique of matched asymptotic expansions $\frac{45}{5}$ and blackfolds $s^{6}$ is based on this picture.

The blackfold approach has led to perturbative solutions for black rings in more than five dimensions, when the ring radius is sufficiently large. ${ }^{4}$ However, it cannot deal with solutions, whose ring radius is not large as compared to the radius of the $S^{D-3}$-sphere. Thus, to obtain the phase diagram of higher-dimensional black rings, numerical methods are called for.

In his talk Eugen Radu presented solutions for balanced black rings in six dimensions, that were obtained numerically, employing two different methods, a finite difference solver and a spectral method ${ }^{7}$ The general picture he unveiled for these black rings exhibits remarkable similarities to the five-dimensional case. In particular, there are again two branches of black ring solutions. The scaled horizon area $a_{\mathrm{H}}$ possesses a cusp at a minimal value of the scaled angular momentum $j$, where it assumes its maximal value. Apart from the region close to the cusp, the thin black ring branch is remarkably well approximated by the blackfold approach.

Starting from the cusp, the branch of fat black rings extends only over a short interval of the scaled angular momentum. In this range there exist three different solutions with the same global charges, violating uniqueness as in five dimensions. This short branch is expected to end in a critical merger configuration $\frac{418}{4}$ Here a branch of 'pinched' black holes should be approached in a horizon topology changing transition. The branch of 'pinched' black holes itself is expected to branch off from a critical Myers-Perry solution, where a Gregory-Laflamme-like instability arises. 9.14 It remains a numerical challenge to obtain the branch of 'pinched' black holes.

Eugen Radu concluded his talk with a discussion of other black hole solutions, amenable to these numerical methods. In particular, he discussed static solutions with horizon topology $S^{2} \times S^{D-4}$ obtained previously ${ }^{15}$ and static composite black objects in more than five dimensions $\frac{16}{16}$ While these static solutions are unbalanced, the inclusion of rotation or of higher curvature terms might balance them. Recently, 
balance of $S^{2} \times S^{D-4}$ solutions could be achieved with a help of an external magnetic field ${ }^{17}$ Thus these solutions approach asymptotically a Melvin universe background.

\subsection{Anti-de Sitter black holes and solitons with scalar hair}

Let us now turn to higher-dimensional black holes in the framework of the AdS/CFT correspondence ${ }^{[18} \mathrm{A}$ main point of interest here has been the description of so-called holographic superconductors with the help of black holes in higher dimensional space-times 1922

As observed by Gubser $\frac{19}{19}$ below a critical temperature electrically charged black holes can become unstable to the formation of scalar hair. This instability results from the fact, that the effective mass of the scalar field can assume negative values below the Breitenlohner-Freedman (BF) bound ${ }^{23 \mid 24}$ close to the black hole horizon. The scalar field then assumes a finite value on the horizon. Its value on the AdS boundary is then associated with a condensate of the boundary field theory. The reason for this behaviour is related to the fact that the scalar field possesses an effective mass that depends on the geometry of the space-time if the scalar field is charged under a $\mathrm{U}(1)$. Close to the horizon the effective mass can drop below the BF bound, while asymptotically it stays well above, ensuring the stability of asymptotic AdS.

An interesting open issue here was the question whether higher curvature corrections can suppress the condensation. In $\frac{25}{25}$ it was shown, that the formation of scalar hair cannot be suppressed in Einstein-Gauss-Bonnet gravity in the "probe limit", where backreaction is neglected. In her talk Betti Hartmann addressed the problem away from the probe limit, based on the self-consistent treatment of the coupled system of Einstein-Gauss-Bonnet-matter equations. ${ }^{26}$

In her study of the formation of scalar hair on electrically charged black holes in five dimensional anti-de Sitter space-time, the Gauss-Bonnet coupling constant was treated as a parameter. The results, however, showed, that - analogous to the probe limit - a critical temperature exists, where condensation sets in. While this critical temperature decreases, as the Gauss-Bonnet coupling constant is increased, it remains positive for any value of this coupling and for arbitrarily large gravitational coupling.26

While her first study ${ }^{26}$ considered planar black holes and a charged scalar field, she subsequently investigated hyperbolic black holes with a neutral scalar field. ${ }^{27}$ Also for these hyperbolic black holes in Einstein-Gauss-Bonnet gravity it was found that within a certain range of the scalar field mass they become unstable to the condensation of the scalar field. Interestingly, a whole family of hairy black hole solutions was found, that could be labeled by the number of nodes of the scalar field function.

In the case of an uncharged scalar field it is not the coupling to the $\mathrm{U}(1)$ gauge field that leads to the condensation, but rather the fact that the near-horizon geometry of a black hole close to extremality possesses in general an $\mathrm{AdS}_{2}$ factor. While 
the mass of the scalar field can be above the BF bound associated with $\mathrm{AdS}_{5}$ asymptotically, it can be below the BF bound associated with the $\mathrm{AdS}_{2}$ of the horizon. Thus the horizon can become unstable with respect to scalar hair formation.

Finally, Betti Hartmann considered charged Gauss-Bonnet black holes with spherical horizon topology ${ }^{28}$ Again an instability related to the condensation of a scalar field was observed. Besides it was seen that hairy Gauss-Bonnet black holes never tend to regular soliton solutions when the horizon radius tends to zero.

\subsection{New solution generation techniques using $S L(2, R)$-duality and general black holes}

To obtain new analytical black hole solutions various solution generation techniques are known. A straightforward method to obtain new black hole solutions in $D$ dimensions is based on the Kaluza-Klein reduction. Here one embeds a $D$-dimensional vacuum solution in $D+1$ dimensions, performs a symmetry transformation, and then reduces the solution to $D$ dimensions ${ }^{29} \sqrt[39]{39}$ In the simplest case, when a single boost is applied, the procedure leads to electrically charged Einstein-Maxwelldilaton black holes in $D$ dimensions, obtained in four dimensions by Chodos and Detweiler ${ }^{31}$ in the static case, and by Frolov et al $\frac{35}{3 n}$ in the rotating case.

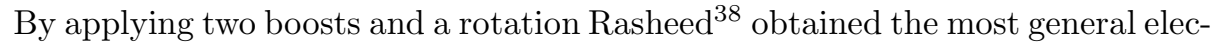
trically and magnetically charged rotating black hole solutions of Einstein-Maxwelldilaton theory in four dimensions. From a five-dimensional perspective, however, it corresponds to a solution of pure Einstein gravity. This solution plays an important role in the construction of new solutions performed by Shinya Tomizawa, as he explained in his talk.

A shown in ${ }^{40}$ the Kaluza-Klein reduction of five-dimensional minimal supergravity to four dimensions yields a theory with a dilaton, an axion, and two gauge fields. The SL $(2, R)$ symmetry of the resulting set of equations of motion was utilized by Shinya Tomizawa $a^{41} 43$ to generate new solutions in five-dimensional minimal supergravity. From the four-dimensional point of view these black hole solutions carry six different global charges. These are the mass, the angular momentum, the KaluzaKlein electric and magnetic charge, and the electric and magnetic charge of the Maxwell field.

The previously known black hole solutions of this theory were static 44,48 with one exception $\stackrel{49}{ }$ Moreover, none of the known solutions did carry all six charges. With the new solution generation technique presented in this talk finally black hole solutions with all six charges could be generated 14143

First the Rasheed solution ${ }^{38}$ represented the seed solution, i.e., the known solution, on which a number of $\mathrm{SL}(2, R)$ transformations were performed to obtain new solutions 41 However, in these new solutions four of the six global charges, the Kaluza-Klein electric and magnetic charge as well as the electric and magnetic charge of the Maxwell field, are connected by a relation. Thus the six global charges of these solutions are not fully independent. 
Subsequently, another seed solution was employed, that corresponds to a boosted rotating electrically and magnetically charged black string solution with five parameters 50 This seed then led to the most general set of black hole solutions of the theory. ${ }^{43}$ These are specified by six independent global charges.

\subsection{New squashed black hole solution with electromagnetic field}

Another interesting type of solution with compact dimensions are the Kaluza-Klein black hole solutions with squashed horizons, where the squashing transformation may also be viewed as a solution generation technique. Squashed black holes in five dimensions were first constructed by Ishihara and Matsuno. ${ }^{46}$ Possessing an $S^{3}$ horizon topology, these black hole solutions asymptote to a locally flat space-time, which is a twisted $S^{1}$ fiber bundle over the four-dimensional Minkowski space-time. Sec $\sqrt{51}$ for an overview of black hole solutions with squashed horizons.

In his talk Stoytcho Yazadjiev first presented a new squashed Kaluza-Klein black hole solution. It represents a solution of Einstein-Maxwell-dilation theory in five dimensions, obtained from a Kaluza-Klein reduction of six-dimensional Einstein gravity. Its various limits represent the Ishihara-Matsuno solution, ${ }^{46}$ and Einstein-Maxwell-dilation generalizations of the dipole black string and the GrossPerry-Sorkin Kaluza-Klein monopole ${ }^{[52153}$ According to the topology theorem $[5455$ the horizon topology of this solution is determined by its interval structure. The uniqueness theorem for Einstein-Maxwell-dilation theory with compact extra dimensions $\sqrt{56157}$ furthermore states, that this solution is fully characterized by its interval structure and by its magnetic fluxes.

With the presentation of his second new solution Stoytcho Yazadjiev turned to Kaluza-Klein multi-black hole configurations (see e.g. the review ${ }^{51}$ ). In particular, he presented a solution describing black lenses in equilibrium in Einstein-Maxwelldilation theory in five dimensions. 58

Interestingly, an old solution by Clement ${ }^{59}$ had been reconsidered recently by Matsuno et al ${ }^{60}$ and interpreted as an equilibrium configuration of extremal rotating black holes, held apart by their repulsive spin-spin interaction. Subsequently, they generalized this solution to five-dimensional Einstein-Maxwell theory and minimal supergravity ${ }^{61}$ The generalization by Stoytcho Yazadjiev, on the other hand, gave a solution that describes several black lenses in equilibrium, where the electric charge of each black lense is zero. Moreover, the horizons are superconducting in the sense that they expel the magnetic flux lines $62[63$ The gravitational force between the black lenses is balanced by the tension of the compact dimension and their repulsive spin-spin interaction.

\subsection{Charged black holes on the Taub-bolt instanton and their thermodynamics}

Besides multi-black hole solutions, also sequences of Kaluza-Klein bubbles and black holes were analyzed in recent years 64 Here the bubbles provide a means to hold 
the black holes apart, allowing for multi-black hole space-times without conical singularites. At the same time, the bubbles may be viewed as the simplest case of gravitational instantons.

Associated with the Gross-Perry-Sorkin magnetic monopole,,$[52[53$ the self-dual Taub-NUT instanton represents another gravitational instanton. The squashed black holes ${ }^{46}$ may be viewed as black holes sitting on this instanton. Further instanton backgrounds are Euclidean Kerr, Taub-bolt or Eguchi-Hanson instantons. Chen and Teo employed the rod-structure formalism to provide a classification of gravitational instantons, ${ }^{71}$ based on the classification theorems. ${ }^{[5 / 56}$ They also provided a recent review on black holes on gravitational instantons ${ }^{72}$

In her talk Petya Nedkova first recalled the partial classifications of gravitational instantons by means of (i) the asymptotic space-time structure $\frac{73}{,}$ and (ii) the fixed point sets of the $\mathrm{U}(1)$ subgroup of the isometry group ${ }^{74}$ as well as the complete classification by means of the fixed point sets of the full $U(1) \times U(1)$ isometry group $\frac{54155}{5 h e n}$ she discussed the generalization obtained by including event horizons, i.e., black holes 'sitting' on gravitational instantons.

Here her main focus was the analysis of the physical properties of these solutions. In particular, extending previous work ${ }^{75}[80$ Petya Nedkova derived the expressions for the mass, the tension, the NUT charge and the NUT potential, and she obtained new Smarr-like relations and the first law for the mass and the tension 81 The culmination was the presentation of a new solution, obtained with the solution generation technique. ${ }^{82}$ The seed solution is a static black hole on a Taub-bolt instanton, $\sqrt{72}$ which is transformed into a charged black hole on the Taub-bolt instanton of Einstein-Maxwell-dilaton theory ${ }^{81}$ The new thermodynamical formulae then yielded the Smarr relations and the first law for this solution.

\subsection{Black rings on Taub-NUT}

The Emparan-Real ${ }^{[2}$ and the Pomeransky-Sen'kov $\sqrt{83}$ black rings are singly and doubly rotating asymptotically flat vacuum solutions. But like black holes, also black rings may be considered on other backgrounds (see e.g! ${ }^{72}$ ). Black rings on bubbles, for instance, were considered by Yazadjiev and Nedkova ${ }^{69 \mid 70}$ In his talk Yu Chen considered vacuum black rings on Taub-NUT backgrounds .84

In contrast to black rings on Taub-NUT backgrounds in supersymmetric theories, $44 \mid 45[85$ vacuum black rings on Taub-NUT backgrounds proved to be more difficult to construct. While the static solutions of Ford et al .86 exhibited conical singularities, in the rotating solutions of Camps 87 balance could be achieved. However, the rotations with respect to the $S^{1}$ and the $S^{2}$ directions of the ring were not independent. It was the goal of Chen and Teo to obtain the most general balanced rotating black rings on Taub-NUT. 84

As explained by $\mathrm{Yu}$ Chen in his talk, their construction of black rings on TaubNUT was based on the inverse scattering method ${ }^{88189} \mathrm{He}$ first discussed the singly rotating black rings on Taub-NUT. These possess the correct rod structure and may 
be viewed as the direct generalizations of the Emparan-Reall black rings, which are recovered in the limit of infinite NUT charge. When balance is imposed, the solutions are completely regular: they possess no naked singularities, no closed timelike curves, no Dirac-Misner string.

It is very interesting to perform a Kaluza-Klein reduction on these black rings along the direction of the Killing vector that generates the finite circle at infinity. Then the NUT of the space-time reduces to the Gross-Perry-Sorkin monopole, and the black ring reduces to an electrically charged black hole. Thus the magnetic charge is carried by the monopole, while the electric charge is carried by the black hole. The angular momentum of the system then arises only from the electromagnetic field, and is given by the product of the electric and the magnetic charge. In general, the monopole and the black hole are separated by a conical singularity, but the reduced four-dimensional system is balanced, when balance was achieved for the five-dimensional solution. 84

$\mathrm{Yu}$ Chen then addressed the Taub-NUT generalizations of the PomeranskySen'kov black rings. In particular, he presented two classes of extremal solutions, where the $S^{2}$ rotation is saturated so that the surface gravity vanishes $\frac{84}{\text { The sign }}$ of the $S^{2}$ rotation distinguishes the two classes, which represent physically different configurations for finite NUT charge. But both classes reduce to the extremal Pomeransky-Sen'kov black rings in the infinite NUT charge limit. A Kaluza-Klein reduction now yielded a purely electrically charged rotating extremal black hole, that can be kept in balance with a magnetic monopole. The extremal black hole is associated with the Rasheed solution. ${ }^{[3]}$ Depending on the sign of the rotation of the $S^{2}$, the black hole may be considered as corotating or counterrotating with respect to the angular momentum carried by the electromagnetic field.

\section{New investigations of the properties of higher-dimensional solutions}

We now turn to the discussion of physical and mathematical properties of higherdimensional black hole solutions, starting with a new approach to address their stability.

\subsection{Black hole instabilities and local Penrose inequalities}

While a stability analysis of higher dimensional black holes is very important from a pure gravity point of view, the gravity/gauge duality presents a further motivation. As discussed above, a stability analysis of AdS black holes can help understand the phase structure of the corresponding boundary field theory.

A linear stability analysis of the static Schwarzschild-Tangherlini black holes showed them to be stable ${ }^{90}$ A stability analysis for rotating higher dimensional black holes, however, is much more involved, since the perturbation equations are partial differential equations, where the separation of variables presents a basic difficulty ${ }^{93}$ So far basically the cohomogeneity-1 case of black holes with equal 


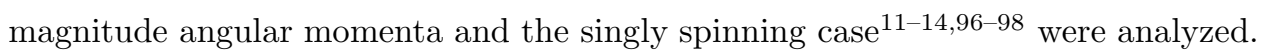
As predicted by Emparan and Myers, $\frac{10}{10}$ the Gregory-Laflamme type instabilities 9 were found, $11+14$ but also bar-mode instabilities were discovered ${ }^{9798}\left(\mathrm{Sec}^{99}\right.$ for a recent review.)

In his talk Keiju Murata presented a new and simpler method to investigate the stability of black holes $\frac{100}{10}$ This method allows to demonstrate instability by employing inequalities analogous to the Penrose inequality (sec ${ }^{101}$ for a recent review). Keiju Murata explained, that if a certain stationary black hole is stable, then initial data describing a small perturbation of the black hole must satisfy a local Penrose inequality, and that a black hole must be unstable if one can find initial data that violates this inequality 100

In the derivation of the Penrose inequality a Taylor expansion for a oneparameter family of solutions is considered. For rotating black holes with axial symmetry the inequality then reads

$$
Q=\ddot{M}(0)-\frac{1}{4} T \ddot{A}_{\text {app }}(0)-\frac{1}{T c_{J}} \dot{M}(0)^{2} \geq 0
$$

where the derivative is with respect to the small parameter, and $M$ denotes the mass, $T$ the temperature, $A_{\text {app }}$ the apparent horizon area and $c_{J}$ the heat capacity at constant angular momentum $\frac{100}{}$ According to Keiju Murata the strategy is then to construct initial data describing a black hole perturbation, to read off the deviation of the mass and the area of apparent horizon, to substitute them into $Q$, and to check the sign. If $Q<0$, the black hole is unstable.

His first application were black strings, where the inequality simplifies to $\ddot{A}_{\text {app }}(0)>0$. Although this technique gives only a sufficient condition for instability, the results are amazingly close to the exact values for the Gregory-Laflamme instability ${ }^{9}$ For singly spinning Myers-Perry black holes, on the other hand, the results are consistent with the numerical results, 11,14 but the instability is seen only at notably higher values of the spin. When applied to black rings, the new method showed, that singly and doubly spinning black rings are unstable on their fat black ring branch!100 The method should prove powerful also in many other cases, and in particular in asymptotically AdS space-times.

\subsection{Existence and some properties of thermodynamic black di-rings}

We now address some properties of multi-black objects. Balanced black Saturns, for instance, are regular stationary solutions ${ }^{102}$ In general, these two-component solutions exhibit a two-fold continuous non-uniqueness. However, by imposing thermodynamic equilibrium on the system, i.e., by requiring that the black hole and the black ring have equal temperature and equal angular velocity, one drastically reduces the phase space ${ }^{103}$ In particular, this selects a single family of solutions, consisting of a thin black Saturn branch and a fat black Saturn branch!103 
Another such composite system are black di-rings. In his poster presentation Takashi Mishima recalled that black di-rings were obtained with two different methods: first, by employing a solution generating technique similar to a Bäcklund transformation, $\frac{104}{10}$ and second, by the inverse scattering method, employing two different seeds 1051106

After clarifying that the black di-rings obtained by the two methods are completely equivalent, $\frac{107}{}$ Takashi Mishima addressed the question, whether black dirings can exist in thermodynamic equilibrium. Indeed, as for the black Saturn, there exists a single family of solutions, consisting of a thin black di-ring branch and a fat black di-ring branch ${ }^{107}$ The fat black di-ring branch ends in a singular configuration at the same point, where the Myers-Perry and black Saturn branches end. The thin black di-ring branch, on the other hand, has a limit, where the ratio of the total area of the black di-ring to that of the black ring becomes one-half.

\subsection{Analyticity of event horizons of extremal Kaluza-Klein black holes}

As argued by Masashi Kimura in his talk, analyticity of the metric is a crucial property for the investigation of the global structure of the space-time. ${ }^{108}$ In particular, he addressed the analyticity of the horizon for extremal Kaluza-Klein multi-black holes.

In four dimensions the Majumdar-Papapetrou solution ${ }^{109 \mid 110}$ describes a set of extremal charged static black holes, where the gravitational and the Coulomb forces exactly balance. These solutions were extended to multi-black holes in higher dimensions by Myers ${ }^{111}$ Whereas the black holes of the Majumdar-Papapetrou solution possess analytic event horizons, 112 the event horizons of the higher-dimensional multi-black holes ${ }^{111}$ are not analytic! $113-115$

Masashi Kimura then considered a toy model for extremal Kaluza-Klein multiblack holes. In particular, he suggested to superpose the black holes periodically and to make identifications, either with respect to the single period $l$ in the case of $S^{1}$, or with respect to all $N$ periods $l^{(n)}$ in the case of $T^{N}$ extra dimensions. He explained that (i) for two black holes the metric is $C^{2}$, but not $C^{3}$ at the horizon in five dimensions, while it is $C^{1}$, but not $C^{2}$ in more than five dimensions; (ii) for $S^{1}$ and $T^{N}$ compactifications the metric is analytic in five dimensions, while it is again $C^{1}$, but not $C^{2}$ in more than five dimensions.

\subsection{Chaos in geodesic motion around a black ring}

To understand the physical properties of black hole solutions it is essential to study the orbits of test particles and light in these space-times. The geodesic equations for test particles and light in Myers-Perry black hole space-times are separable.116 118 Geodesics in Myers-Perry black hole space-times were studied in $119 \llbracket 122$

In his talk Takahisa Igata addressed the geodesics of black ring space-times, where separability does not appear to hold in general. Employing ring coordinates, 
the equations of motion could be separated only in special cases. These correspond to either geodesics on the two rotational axes, or to zero energy null geodesics, which can only exist in the ergosphere ${ }^{123 \mid 124}$ In these special cases the geodesic motion was studied numerically and analytically in the singly spinning black ring spacetimes, $\frac{123[125,128}{12}$ as well as in the doubly spinning black ring space-times. 124129 Interestingly, in contrast to Myers-Perry black hole space-times, there are stable bound orbits in black ring space-times.126/128 130

Separability of the Hamilton-Jacobi equation depends on the coordinate system. Therefore one might try to think of a coordinate system in which it would be possible to separate the Hamilton-Jacobi equation for general geodesics. To settle the issue, Takahisa Igata analyzed the geometry of the singly spinning black rings further in his talk. By using the Poincaré map he argued that these ring space-times possess chaotic bound orbits 131 The appearance of chaos, however, implies that there is no additional constant of motion in the black ring metric. Thus it should not be possible to separate the Hamilton-Jacobi equation of singly spinning black rings in any coordinate system, in general. It will be interesting to see whether chaotic motion will also appear in the doubly spinning black ring space-times.

\section{Conclusion}

The field is still very active and attracting many excellent young researchers, as seen in this and related sessions. Clearly, higher-dimensional black objects are still in the focus of the attention of the community.

\section{References}

1. R. C. Myers and M. J. Perry, Annals Phys. 172, 304 (1986).

2. R. Emparan and H. S. Reall, Phys. Rev. Lett. 88, 101101 (2002) arXiv:hep-th/0110260.

3. R. Emparan and H. S. Reall, Living Rev. Rel. 11, 6 (2008) arXiv:0801.3471 [hepth]].

4. R. Emparan, T. Harmark, V. Niarchos, N. A. Obers and M. J. Rodriguez, JHEP 0710 (2007) 110 arXiv:0708.2181 [hep-th]].

5. R. Emparan, T. Harmark, V. Niarchos and N. A. Obers, JHEP 1004, 046 (2010) arXiv:0912.2352 [hep-th]];

6. R. Emparan, T. Harmark, V. Niarchos and N. A. Obers, Phys. Rev. Lett. 102 (2009) 191301 arXiv:0902.0427 [hep-th]].

7. B. Kleihaus, J. Kunz and E. Radu, Phys. Lett. B 718, 1073 (2013) arXiv:1205.5437 [hep-th]].

8. R. Emparan and P. Figueras, JHEP 1011 (2010) 022 arXiv:1008.3243 [hep-th]].

9. R. Gregory and R. Laflamme, Phys. Rev. Lett. 70, 2837 (1993) arXiv:hep-th/9301052

10. R. Emparan and R. C. Myers, JHEP 0309, 025 (2003) hep-th/0308056.

11. O. J. C. Dias, P. Figueras, R. Monteiro, J. E. Santos and R. Emparan, Phys. Rev. D 80 (2009) 111701 arXiv:0907.2248 [hep-th]].

12. O. J. C. Dias, P. Figueras, R. Monteiro, H. S. Reall and J. E. Santos, JHEP 1005, 076 (2010) arXiv:1001.4527 [hep-th]]. 
13. O. J. C. Dias, P. Figueras, R. Monteiro and J. E. Santos, Phys. Rev. D 82, 104025 (2010) arXiv:1006.1904 [hep-th]].

14. O. J. C. Dias, R. Monteiro and J. E. Santos, JHEP 1108, 139 (2011) arXiv:1106.4554 [hep-th]].

15. B. Kleihaus, J. Kunz and E. Radu, Phys. Lett. B 678, 301 (2009) arXiv:0904.2723 [hep-th]].

16. B. Kleihaus, J. Kunz, E. Radu and M. J. Rodriguez, JHEP 1102, 058 (2011) arXiv:1010.2898 [gr-qc]].

17. B. Kleihaus, J. Kunz and E. Radu, Phys. Lett. B 723, 182 (2013) arXiv:1303.2190 [gr-qc]].

18. J. M. Maldacena, Adv. Theor. Math. Phys. 2, 231 (1998) hep-th/9711200.

19. S. S. Gubser, Phys. Rev. D 78, 065034 (2008) arXiv:0801.2977 [hep-th]].

20. S. A. Hartnoll, C. P. Herzog and G. T. Horowitz, Phys. Rev. Lett. 101, 031601 (2008) arXiv:0803.3295 [hep-th]].

21. S. A. Hartnoll, C. P. Herzog and G. T. Horowitz, JHEP 0812, 015 (2008) arXiv:0810.1563 [hep-th]].

22. G. T. Horowitz and M. M. Roberts, Phys. Rev. D 78, 126008 (2008) arXiv:0810.1077 [hep-th]].

23. P. Breitenlohner and D. Z. Freedman, Phys. Lett. B 115, 197 (1982).

24. P. Breitenlohner and D. Z. Freedman, Annals Phys. 144, 249 (1982).

25. R. Gregory, S. Kanno and J. Soda, JHEP 0910, 010 (2009) arXiv:0907.3203 [hepth]].

26. Y. Brihaye and B. Hartmann, Phys. Rev. D 81, 126008 (2010) arXiv:1003.5130 [hep-th]].

27. Y. Brihaye and B. Hartmann, Phys. Rev. D 84, 084008 (2011) arXiv:1107.3384 [gr-qc]].

28. Y. Brihaye and B. Hartmann, Phys. Rev. D 85, 124024 (2012) arXiv:1203.3109 [gr-qc]].

29. D. Maison, Gen. Rel. Grav. 10, 717 (1979).

30. D. Maison, Lect. Notes Phys. 540, 273 (2000).

31. A. Chodos and S. L. Detweiler, Gen. Rel. Grav. 14, 879 (1982).

32. P. Dobiasch and D. Maison, Gen. Rel. Grav. 14, 231 (1982).

33. G. W. Gibbons and D. L. Wiltshire, Annals Phys. 167, 201 (1986) [Erratum-ibid. 176, 393 (1987)].

34. G. W. Gibbons and K. -i. Maeda, Nucl. Phys. B 298, 741 (1988).

35. V. P. Frolov, A. I. Zelnikov and U. Bleyer, Annalen Phys. 44, 371 (1987).

36. P. Breitenlohner, D. Maison and G. W. Gibbons, Commun. Math. Phys. 120, 295 (1988).

37. J. H. Horne and G. T. Horowitz, Phys. Rev. D 46, 1340 (1992) hep-th/9203083.

38. D. Rasheed, Nucl. Phys. B 454, 379 (1995) hep-th/9505038.

39. J. Kunz, D. Maison, F. Navarro-Lerida and J. Viebahn, Phys. Lett. B 639, 95 (2006) hep-th/0606005.

40. A. H. Chamseddine and H. Nicolai, Phys. Lett. B 96, 89 (1980).

41. S. Mizoguchi and S. Tomizawa, Phys. Rev. D 84, 104009 (2011) arXiv:1106.3165 [hep-th]].

42. S. Mizoguchi and S. Tomizawa, Phys. Rev. D 86, 024022 (2012) arXiv:1201.3063 [hep-th]].

43. S. Tomizawa and S. Mizoguchi, Phys. Rev. D 87, 024027 (2013) arXiv:1210.6723 [hep-th]].

44. H. Elvang, R. Emparan, D. Mateos and H. S. Reall, JHEP 0508, 042 (2005) 
hep-th/0504125.

45. D. Gaiotto, A. Strominger and X. Yin, JHEP 0602, 023 (2006) hep-th/0504126.

46. H. Ishihara and K. Matsuno, Prog. Theor. Phys. 116, 417 (2006) hep-th/0510094.

47. T. Nakagawa, H. Ishihara, K. Matsuno and S. Tomizawa, Phys. Rev. D 77, 044040 (2008) arXiv:0801.0164 [hep-th]].

48. S. Tomizawa, H. Ishihara, K. Matsuno and T. Nakagawa, Prog. Theor. Phys. 121, 823 (2009) arXiv:0803.3873 [hep-th]].

49. S. Tomizawa, Y. Yasui and Y. Morisawa, Class. Quant. Grav. 26, 145006 (2009) arXiv:0809.2001 [hep-th]].

50. G. Compere, S. de Buyl, S. Stotyn and A. Virmani, JHEP 1011, 133 (2010) arXiv:1006.5464 [hep-th]].

51. S. Tomizawa and H. Ishihara, Prog. Theor. Phys. Suppl. 189, 7 (2011) arXiv:1104.1468 [hep-th]].

52. D. J. Gross and M. J. Perry, Nucl. Phys. B 226, 29 (1983).

53. R. d. Sorkin, Phys. Rev. Lett. 51, 87 (1983).

54. S. Hollands and S. Yazadjiev, Commun. Math. Phys. 283, 749 (2008) arXiv:0707.2775 [gr-qc]].

55. S. Hollands and S. Yazadjiev, Class. Quant. Grav. 25, 095010 (2008) arXiv:0711.1722 [gr-qc]].

56. S. Hollands and S. Yazadjiev, Commun. Math. Phys. 302, 631 (2011) arXiv:0812.3036 [gr-qc]].

57. S. S. Yazadjiev, Phys. Rev. D 82, 024015 (2010) arXiv:1002.3954 [hep-th]].

58. S. S. Yazadjiev, Phys. Rev. D 86, 107504 (2012) arXiv:1209.3488 [gr-qc]].

59. G. Clement, Gen. Rel. Grav. 18, 861 (1986).

60. K. Matsuno, H. Ishihara, M. Kimura and T. Tatsuoka, Phys. Rev. D 86, 044036 (2012) arXiv:1206.4818 [hep-th]].

61. K. Matsuno, H. Ishihara, M. Kimura and T. Tatsuoka, Phys. Rev. D 86, 104054 (2012) arXiv:1208.5536 [hep-th]].

62. J. Bicak and L. Dvorak, Phys. Rev. D 22, 2933 (1980).

63. A. Chamblin, R. Emparan and G. W. Gibbons, Phys. Rev. D 58, 084009 (1998) hep-th/9806017.

64. H. Elvang and G. T. Horowitz, Phys. Rev. D 67 (2003) 044015 arXiv:hep-th/0210303.

65. H. Elvang, T. Harmark and N. A. Obers, JHEP 0501 (2005) 003 arXiv:hep-th/0407050.

66. S. Tomizawa, H. Iguchi and T. Mishima, Phys. Rev. D 78, 084001 (2008) hep-th/0702207 [HEP-TH]].

67. J. Kunz and S. Yazadjiev, Phys. Rev. D 79, 024010 (2009) arXiv:0811.0730 [hepth]].

68. S. S. Yazadjiev and P. G. Nedkova, Phys. Rev. D 80, 024005 (2009) arXiv:0904.3605 [hep-th]].

69. S. S. Yazadjiev and P. G. Nedkova, JHEP 1001, 048 (2010) arXiv:0910.0938 [hepth]].

70. P. G. Nedkova and S. S. Yazadjiev, Phys. Rev. D 82, 044010 (2010) arXiv:1005.5051 [hep-th]].

71. Y. Chen and E. Teo, Nucl. Phys. B 838, 207 (2010) arXiv:1004.2750 [gr-qc]].

72. Y. Chen and E. Teo, Nucl. Phys. B 850, 253 (2011) arXiv:1011.6464 [hep-th]].

73. G. W. Gibbons, C. N. Pope and H. Romer, Nucl. Phys. B 157, 377 (1979).

74. G. W. Gibbons and S. W. Hawking, Commun. Math. Phys. 66, 291 (1979).

75. R. B. Mann and C. Stelea, Phys. Lett. B 634, 531 (2006) hep-th/0511180. 
76. D. Astefanesei and E. Radu, Phys. Rev. D 73, 044014 (2006) hep-th/0509144.

77. P. K. Townsend and M. Zamaklar, Class. Quant. Grav. 18, 5269 (2001) hep-th/0107228.

78. J. H. Traschen and D. Fox, Class. Quant. Grav. 21, 289 (2004) gr-qc/0103106.

79. J. H. Traschen, Class. Quant. Grav. 21, 1343 (2004) hep-th/0308173.

80. D. Kastor, S. Ray and J. Traschen, Class. Quant. Grav. 25, 125004 (2008) arXiv:0803.2019 [hep-th]].

81. P. G. Nedkova and S. S. Yazadjiev, Phys. Rev. D 84, 124040 (2011) arXiv:1109.2838 [hep-th]].

82. S. S. Yazadjiev, hep-th/0507097.

83. A. A. Pomeransky and R. A. Sen'kov, hep-th/0612005

84. Y. Chen and E. Teo, JHEP 1206, 068 (2012) arXiv:1204.3116 [hep-th]].

85. I. Bena, P. Kraus and N. P. Warner, Phys. Rev. D 72, 084019 (2005) hep-th/0504142.

86. J. Ford, S. Giusto, A. Peet and A. Saxena, Class. Quant. Grav. 25, 075014 (2008) arXiv:0708.3823 [hep-th]].

87. J. Camps, R. Emparan, P. Figueras, S. Giusto and A. Saxena, JHEP 0902, 021 (2009) arXiv:0811.2088 [hep-th]].

88. V. Belinski and E. Verdaguer, Gravitational solitons, Cambridge University Press, U.K (2001).

89. A. A. Pomeransky, Phys. Rev. D 73, 044004 (2006) hep-th/0507250.

90. H. Kodama and A. Ishibashi, Prog. Theor. Phys. 110, 701 (2003) arXiv:hep-th/0305147.

91. A. Ishibashi and H. Kodama, Prog. Theor. Phys. 110, 901 (2003) arXiv:hep-th/0305185.

92. R. A. Konoplya and A. Zhidenko, Nucl. Phys. B 777, 182 (2007) arXiv:hep-th/0703231.

93. H. K. Kunduri, J. Lucietti and H. S. Reall, Phys. Rev. D 74, 084021 (2006) arXiv:hep-th/0606076.

94. K. Murata and J. Soda, Class. Quant. Grav. 25, 035006 (2008) arXiv:0710.0221 [hep-th]].

95. K. Murata and J. Soda, Prog. Theor. Phys. 120, 561 (2008) arXiv:0803.1371 [hepth]].

96. H. Kodama, R. A. Konoplya and A. Zhidenko, Phys. Rev. D 81, 044007 (2010) arXiv:0904.2154 [gr-qc]].

97. M. Shibata and H. Yoshino, Phys. Rev. D 81, 021501 (2010) arXiv:0912.3606 [grqc]].

98. M. Shibata and H. Yoshino, Phys. Rev. D 81, 104035 (2010) arXiv:1004.4970 [gr$\mathrm{qc}]]$.

99. K. Murata, Prog. Theor. Phys. Suppl. 189, 210 (2011).

100. P. Figueras, K. Murata and H. S. Reall, Class. Quant. Grav. 28, 225030 (2011) arXiv:1107.5785 [gr-qc]].

101. M. Mars, Class. Quant. Grav. 26, 193001 (2009) arXiv:0906.5566 [gr-qc]].

102. H. Elvang and P. Figueras, JHEP 0705 (2007) 050 arXiv:hep-th/0701035.

103. H. Elvang, R. Emparan and P. Figueras, JHEP 0705, 056 (2007) hep-th/0702111.

104. H. Iguchi and T. Mishima, Phys. Rev. D 75 (2007) 064018 arXiv:hep-th/0701043.

105. J. Evslin and C. Krishnan, Class. Quant. Grav. 26 (2009) 125018 arXiv:0706.1231 [hep-th].

106. H. Iguchi, K. Izumi and T. Mishima, Prog. Theor. Phys. Suppl. 189, 93 (2011) arXiv:1106.0387 [gr-qc]]. 
107. H. Iguchi and T. Mishima, Phys. Rev. D 82, 084009 (2010) arXiv:1008.4290 [hepth]].

108. M. Kimura, Phys. Rev. D 78, 047504 (2008) arXiv:0805.1125 [gr-qc]].

109. S. D. Majumdar, Phys. Rev. 72, 390 (1947).

110. A. Papaetrou, Proc. Roy. Irish Acad. (Sect. A) A 51, 191 (1947).

111. R. C. Myers, Phys. Rev. D 35, 455 (1987).

112. J. B. Hartle and S. W. Hawking, Commun. Math. Phys. 26, 87 (1972).

113. G. W. Gibbons, G. T. Horowitz and P. K. Townsend, Class. Quant. Grav. 12, 297 (1995) hep-th/9410073.

114. D. L. Welch, Phys. Rev. D 52, 985 (1995) hep-th/9502146.

115. G. N. Candlish and H. S. Reall, Class. Quant. Grav. 24, 6025 (2007) arXiv:0707.4420 [gr-qc]].

116. D. Kubiznak and V. P. Frolov, Class. Quant. Grav. 24, F1 (2007) gr-qc/0610144.

117. D. N. Page, D. Kubiznak, M. Vasudevan and P. Krtous, Phys. Rev. Lett. 98, 061102 (2007) hep-th/0611083.

118. V. P. Frolov, P. Krtous and D. Kubiznak, JHEP 0702, 005 (2007) hep-th/0611245.

119. V. P. Frolov and D. Stojkovic, Phys. Rev. D 68, 064011 (2003) gr-qc/0301016].

120. C. Gooding and A. V. Frolov, Phys. Rev. D 77, 104026 (2008) arXiv:0803.1031 [gr-qc]].

121. E. Hackmann, V. Kagramanova, J. Kunz and C. Lämmerzahl, Phys. Rev. D 78, 124018 (2008) [Erratum-ibid. 79, 029901 (2009)] arXiv:0812.2428 [gr-qc]].

122. V. Kagramanova and S. Reimers, Phys. Rev. D 86, 084029 (2012) arXiv:1208.3686 [gr-qc]].

123. J. Hoskisson, Phys. Rev. D 78, 064039 (2008) arXiv:0705.0117 [hep-th]].

124. M. Durkee, Class. Quant. Grav. 26, 085016 (2009) arXiv:0812.0235 [gr-qc]].

125. H. Elvang, R. Emparan and A. Virmani, JHEP 0612, 074 (2006) hep-th/0608076.

126. T. Igata, H. Ishihara and Y. Takamori, Phys. Rev. D 82, 101501 (2010) arXiv:1006.3129 [hep-th]].

127. J. Armas, Class. Quant. Grav. 28, 235014 (2011) arXiv:1011.5618 [hep-th]].

128. S. Grunau, V. Kagramanova, J. Kunz and C. Lämmerzahl, Phys. Rev. D 86, 104002 (2012) arXiv:1208.2548 [gr-qc]].

129. S. Grunau, V. Kagramanova and J. Kunz, Phys. Rev. D 87, 044054 (2013) arXiv:1212.0416 [gr-qc]].

130. T. Igata, H. Ishihara and Y. Takamori, Phys. Rev. D 87, 104005 (2013) arXiv:1302.0291 [hep-th]].

131. T. Igata, H. Ishihara and Y. Takamori, Phys. Rev. D 83, 047501 (2011) arXiv:1012.5725 [hep-th]]. 